\title{
Encysted Fluid Collections after Catheter Removal for Peritonitis in Peritoneal Dialysis Patients
}

\author{
Elwaleed A M Elhassan ${ }^{\mathrm{a}^{*}}$, Maha A Hummeida ${ }^{\mathrm{a}}$, Osman-mahir M A Mahir ${ }^{\mathrm{a}}$, Hisham M Alia, Aymun I \\ Mobarak $^{\mathrm{a}}$, Jamal Abdel-Raheem ${ }^{\mathrm{a}}$, Um-Alhasan Mohamed ${ }^{\mathrm{a}}$, Hassan A Musa ${ }^{\mathrm{b}}$ and Hasan Abu-Aisha ${ }^{\mathrm{a}}$
}

a. Ribat center for Regular Peritoneal Dialysis, Ribat University Hospital

b. Department of Microbiology, Ribat University Hospital, Burri, Khartoum, Sudan

\begin{abstract}
Introduction: Peritonitis is a frequent complication of continuous ambulatory peritoneal dialysis (CAPD). This case series describes episodes of peritoneal dialysis (PD) related fungal or Pseudomonas peritonitis that were complicated by the formation of encysted intra abdominal fluid collections despite prompt catheter removal and appropriate therapy.
\end{abstract}

Case series: The first patient presented with fever and abdominal pain two weeks after removing his PD catheter because of fungal peritonitis. Examination revealed fluid collection in the former catheter tunnel. This turned out to be pus on surgical drainage. Abdominal computed tomography (CT) scan revealed another extensive encysted extra-peritoneal fluid collection that was aspirated and found to be clear transudate fluid. The second patient presented with abdominal pain and distention seven weeks after removing her PD catheter because of fungal peritonitis. She had a large intra abdominal encysted fluid collection that was drained under ultra sound guidance. The third patient presented with painless abdominal distention one month after removing the PD catheter because of Pseudomonas peritonitis. He had two encysted extra peritoneal fluid collections that were aspirated under ultra sound guidance. This occurred despite three weeks of treatment with oral fluconazole in the case of the first two patients and appropriate antibiotic therapy in the case of the third patient.

Conclusion: In fungal and Pseudomonas peritonitis, other measures besides immediate initiation of appropriate therapy and prompt removal of the catheter may be necessary to prevent such late complications. Suggested measures include continued intraperitoneal antibiotics by a fresh PD catheter, in addition to systemic antifungal treatment

* Corresponding Author; Ribat Center for Regular Peritoneal Dialysis, Ribat University Hospital, Burri, P.O. Box 55, Khartoum, Sudan. Email: waleedali@yahoo.com
Key Words: CAPD, Fungal peritonitis, Pseudomonas peritonitis, Encysted abdominal fluid collection, Sudan

\section{Introduction}

Peritonitis has been one of the most frequently encountered complications of continuous ambulatory peritoneal dialysis (CAPD) since it was introduced in the Sudan in June 2005. By the end of January 2007 the peritonitis rate for the whole program was 1 episode per 14 patient-months (0.87 per year at risk) [1].

Fungal infection is an uncommon cause of peritonitis in patients on CAPD; however, it carries a higher morbidity and mortality than bacterial infections. The incidence of fungal peritonitis varies between centers, with a reported incidence of 3.9 to $6 \%$ among all PD related peritonitis episodes [1-2]. Reported complications include sclerosing peritonitis, adhesions with resulting bowel obstruction or stricture, invasion of the bowel wall and abscess formation [3].

Tapson et al described the clinical and microbiological features of 10 cases of fungal peritonitis; although all patients survived, morbidity was high. Abscess and adhesion formation were particular problems. Only two patients were able to return to CAPD after microbiological cure [4]. Digenis et al also reported four cases of abdominal abscess complicating peritonitis [5].

Pseudomonas aeruginosa peritonitis, similar to Staphylococcus aureus peritonitis, is generally severe and is often related to a catheter infection. It may also be complicated by severe systemic manifestations, such as digital necrosis [6]. If catheter infection is present or has preceded peritonitis, catheter removal is necessary as per the International Society for PD (ISPD) guidelines [7].

By the end of the first year of operation of the Sudan National PD program the culture negative peritonitis rate was $53 \%$, while Pseudomonas species was responsible for $13.3 \%$ of all episodes [1]. 
Figure 1: Abdominal CT scan of the first patient showing a rim of fluid collection in the anterior abdominal wall

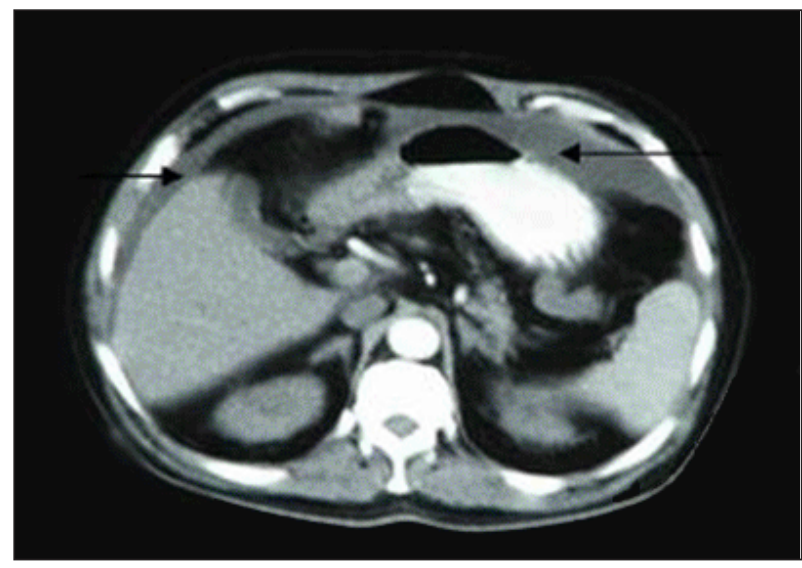

This case series from Ribat PD center, the head-quarters for the seven centers constituting the Sudan National Peritoneal Dialysis Program, describes three episodes of intra-abdominal encysted fluid collections complicating catheter removal for different types of peritonitis.

\section{Case 1}

The first patient was a 66 year old male with long standing diabetes mellitus and end stage renal disease (ESRD) due to diabetic nephropathy who had been on CAPD for about 21 months. He got his first episode of peritonitis six months after commencing CAPD; thereafter, he contracted several episodes of culture negative peritonitis for which he received multiple courses of empirical intraperitoneal (IP) antibiotics according to the ISPD guidelines.

Two weeks after the last episode of culture negative peritonitis, he presented with yet another peritonitis episode that proved to be fungal in origin. He was immediately started on oral Fluconazole $200 \mathrm{mg}$ daily for 3 weeks. The PD catheter was removed on the second day by surgical dissection, the peritoneum, as well as the abdominal wall was closed in layers, and the patient was shifted to hemodialysis (HD).

Two weeks following catheter removal, he presented again with high grade fever, abdominal pain, and purulent discharge from the old exit site. An abdominal computed tomography (CT) scan revealed two separate intra abdominal fluid collections; the first was a localized collection of thick fluid in the old catheter tunnel draining by the exit site, and the second was an encysted fluid collection located in the anterior abdominal wall and extending into both lumbar and iliac fossae (Figure 1).

The purulent collection at the old tunnel site was surgically drained. Ultra sound guided aspiration of the
Figure 2: Abdominal CT scan of the second patient showing a large encysted fluid collection

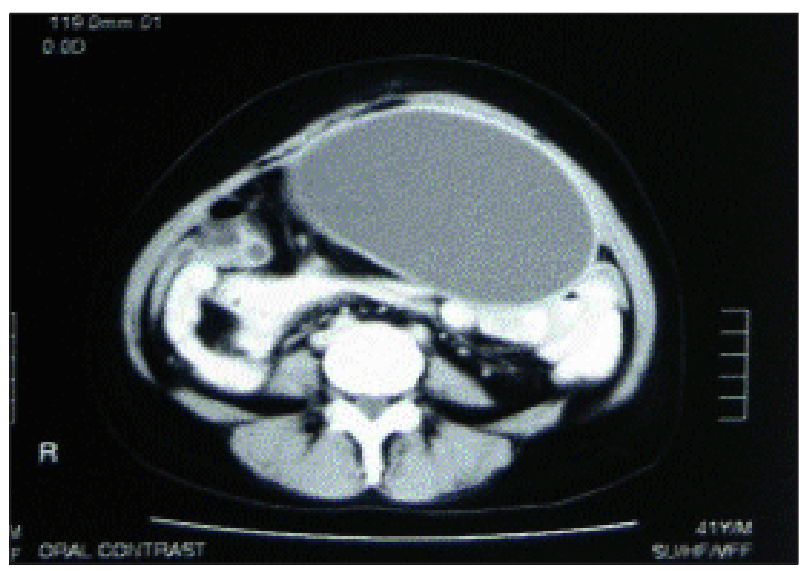

encysted fluid in the anterior abdominal wall revealed a clear yellow transudate with a total white cell count of 50 cells per cubic millimeter, the culture of this fluid revealed no organisms. It was decided to manage the patient conservatively with careful follow up and serial ultra sound scans.

Up to the time of writing this report, several months after the incident, the fluid remains static in size and consistency, causing no troublesome symptoms.

\section{Case 2}

The second patient is a 42 year old female who had been on CAPD for about nine months. She was transferred from HD after a failed arterio-venous fistula and difficult vascular access. She contracted her first peritonitis episode three weeks after starting CAPD.

Following the treatment of the second peritonitis episode she presented complaining of waxing and waning abdominal pain and diarrhea, with abdominal tenderness and guarding despite clear peritoneal effluent. She also had persistently low serum potassium $(2.1 \mathrm{mmol} / \mathrm{l})$ which raised the suspicion of sub acute intestinal obstruction. She was managed conservatively with parenteral potassium supplements and nil orally for a few days, followed by gradual introduction of soft foods. She showed some improvement but continued to suffer from intermittent abdominal discomfort.

Five months later she presented with exit site infection for which she received an oral empirical antibiotic. One week after that she presented with worsening abdominal pain and cloudy peritoneal effluent; and peritoneal fluid analysis revealed Candida species. She was immediately started on Fluconazole $200 \mathrm{mg}$ daily for 3 weeks, the PD catheter was removed four days later by surgical dissection, the peritoneum, as well as the abdominal 
wall was closed in layers, and the patient was shifted to hemodialysis (HD).

Seven weeks later she presented with painless abdominal swelling, she was afebrile and otherwise feeling well. Abdominal CT scan reported a large encysted fluid collection with an air fluid level that was extending from the right subdiaphragmatic area down to the left iliac fossa; the wall was thick and enhancing (Figure 2). Aspiration under ultra sound guidance revealed a large amount of thick fluid, but complete drainage was not feasible. Fluid cultures grew no organisms. After drainage, the patient's symptoms largely abated; subsequent ultrasound scans showed some remaining fluid and the patient is now under close surveillance.

\section{Case 3}

The third case is a 73 year old male with dilated ischemic cardiomyopathy who had been on CAPD for about six months. CAPD was his initial renal replacement therapy. He was referred to us with peritonitis from another center where the Tenckoff catheter was inserted and CAPD commenced four months earlier. Upon referral, he had extrusion of the external catheter cuff which was totally shaved. Culture of the effluent revealed Klebsiella species that was successfully treated with intra peritoneal (IP) antibiotics according to sensitivity results for three weeks. A Few days later he experienced fluid leak from the exit site. This was managed successfully with low fill volumes in the supine position. Several weeks later he self-treated himself with a three day course of IP antibiotics for an episode of cloudy effluent without informing the center.

After that, he presented with frank clinical peritonitis due to Pseudomonas species. This episode was most likely catheter related, but this was not microbiologically confirmed. The catheter was therefore removed on the fourth day by surgical dissection, the peritoneum, as well as the abdominal wall was closed in layers, and the patient was shifted to hemodialysis (HD). He was treated by two antibiotics according to sensitivity results.

About a month later, he presented with a painless superficial abdominal swelling. Ultra sound scan reported left lumbar and left iliac fossa encysted extra-peritoneal fluid collections. Complete aspiration was successful. Microbiological analysis of the aspirate showed a total white blood cell count of 50 cells per cubic millimeter and no organism was isolated. The patient has been symptomfree and stable on HD for the past two months.

The data of the three cases of encysted abdominal fluid collection complicating catheter removal for peritonitis are summarized in (table 1).

\section{Discussion}

In this report we described encysted intra abdominal fluid collections that developed in three patients on CAPD: two after episodes of Candida peritonitis and the third after an episode of Pseudomonas peritonitis. This happened despite early initiation of appropriate therapy and prompt removal of the catheter in each case.

Regarding peritonitis risk factors, all three patients received multiple courses of antibiotics for several prior episodes of peritonitis during their PD course. This concurs with other reports of fungal peritonitis being frequently preceded by antibiotic treatment [2-3]. In addition, the second patient had a recent exit site infection and the third patient had extrusion of the external cuff and fluid leak from the exit site prior to the diagnosis of peritonitis.

The clinical presentation was variable. The first patient presented with abdominal pain, abdominal distension and fever, while the other two presented only with abdominal distension and discomfort. Laboratory constraints precluded recognition of subtypes of the causative organisms, but all patients received appropriate antimicrobial therapy according to the ISPD guidelines and culture and sensitivity results.

Among patients undergoing $\mathrm{PD}$, peritonitis resulting from fungi carries a higher morbidity and mortality than bacterial infections. Reported complications include sclerosing peritonitis, adhesions with resulting bowel obstructions or stricture, invasion of the bowel wall, and abscess formation. Pseudomonas infections are often difficult to eradicate, are associated with catheter infection, and, in severe cases, may result in damage to the peritoneal membrane. Immediate catheter removal is recommended for fungal peritonitis per se, and for Pseudomonas peritonitis complicating an exit site infection [6].

The occurrence of this complication despite immediate initiation of appropriate therapy and prompt removal of the catheter suggests that catheter removal for such cases may need to be accompanied by adequate lavage, and possibly leaving an intra abdominal drain.

Another potentially sound approach is to keep the peritoneum wet by inserting a temporary peritoneal catheter and resuming PD while using IP antibiotics, in addition to systemic antifungal treatment [8]. This approach has resulted in preservation of the peritoneum and increasing the chances of reintroducing the Tenckhoff catheter successfully as described by Keogh et al [8].

If fluid collection proves to be infected, open surgical drainage will probably be more effective than aspiration. 
On the other hand, encysted fluids of transudate nature will probably do well on simple ultrasound guided needle aspirations as shown by our cases .

\section{Conclusion}

Other measures besides immediate initiation of appropriate therapy and prompt removal of the catheter may be necessary to prevent such late complications of CAPD related peritonitis. Suggested measures include continued IP antibiotics by a fresh PD catheter, in addition to systemic antifungal treatment

\section{References}

1. Elhassan EAM, Kaballo B, Fedail H, Abdelraheem MB, Ali T, Medani S, Tammam L, Basheir I, Taha A, Mandour M, El Awad K, Abu-Aisha H. et al. Peritoneal Dialysis in the Sudan. Perit Dial Int. 2007; 27:503-510

2. Wojtaszek E, Ostrowski G, Niemczyk S, Lange J, Grzejszczak A,Matuszkiewicz-Rowinska J. [Fungal peritonitis in patients on peritoneal dialysis] [abstract]. Pol Arch Med Wewn 2006 Jul;116(1):678-82. PMID: 17340975.

3. Das R, Vaux, Barker L, Naik R. Fungal peritonitis complicating peritoneal dialysis: report of 18 cases and analysis of outcomes. Adv Perit Dial. 2006;22:55-9.
4. Uchida M, Sakemi T, Nagano Y, Mizuguchi M. Rectal stenosis associated with fungal peritonitis: a complication of continuous ambulatory peritoneal dialysis. Nephron. 1989;53(3):288-9.

5. Tapson JS, Mansy H, Freeman R. The high morbidity of CAPD fungal peritonitis: description of 10 cases and review of treatment strategies. Q J Med. 1986;61:104753.

6. Digenis GE, Abraham G, Savin E, Blake P, Dombros N, Sombolos K, Vas S, Mathews R, Oreopoulos DG. Peritonitis related deaths in continuous ambulatory peritoneal dialysis (CAPD) patients. Perit Dia Int. 1990;10:45-7.

7. Vassa N, Nolph KD, Khanna R. Pseudomonas peritonitis with white blood cell capillary margination and distal digital necrosis in a patient on CAPD. Perit Dial Int. 1992;12(3):323-5.

8. Piraino B, Bailie GR, Bernardini J, Boeschoten E, Gupta A, Holmes C, Kuijper EJ, Li PK, Lye WC, Mujais S, Paterson DL, Fontan MP, Ramos A, Schaefer F, Uttley L. Peritoneal dialysis-related infections recommendations: 2005 update. Perit Dial Int. 2005 Mar-Apr;25(2):107-31.

9. Keogh JAB, Carr ME, Murray F, McEvoy M, Grant G, Keane CT. Treatment of fungal peritonitis in CAPD patients using peritoneal lavage. Perit dial Int. 5(1) 67-69 1985 